\title{
A duality result between the minimal surface equation and the maximal surface equation
}

\author{
LUIS J. ALÍAS ${ }^{1}$ and BENNETT PALMER ${ }^{2}$ \\ ${ }^{1}$ Departamento de Matemáticas, Universidad de Murcia \\ E-30100 Espinardo, Murcia, Spain \\ ${ }^{2}$ Department of Mathematical Sciences, University of Durham \\ Durham DH1 3LE, England
}

Manuscript received on January 24, 2001; accepted for publication on January 25, 2001;

presented by J. LUCAS BARBOSA

\begin{abstract}
In this note we show how classical Bernstein's theorem on minimal surfaces in the Euclidean space can be seen as a consequence of Calabi-Bernstein's theorem on maximal surfaces in the Lorentz-Minkowski space (and viceversa). This follows from a simple but nice duality between solutions to their corresponding differential equations.
\end{abstract}

Key words: Minimal surface equation, Maximal surface equation, Bernstein's theorem, CalabiBernstein's theorem.

\section{INTRODUCTION}

A minimal surface in Euclidean space $\mathbb{R}^{3}$ is a surface with zero mean curvature. Bernstein (19151917) proved that the planes are the only minimal entire graphs in $\mathbb{R}^{3}$.

THEOREM 1. (BERNSTEIN's THEOREM). The only entire solutions to the minimal surface equation

$$
\operatorname{Minimal}[u]=\operatorname{Div}\left(\frac{D u}{\sqrt{1+|D u|^{2}}}\right)=0
$$

are affine functions.

On the other hand, a maximal surface in the Lorentz-Minkowski space $\mathbb{L}^{3}$ is a spacelike surface with zero mean curvature. Here by spacelike we mean that the induced metric from the Lorentzian

E-mail: ljalias@um.es / bennett.palmer@durham.ac.uk 
metric in $\mathbb{L}^{3}$ is a Riemannian metric on the surface. Calabi (1970) obtained the corresponding version of Bernstein's theorem for the case of maximal surfaces.

THEOREM 2. (CALABI-BERNSTEIN'S THEOREM). The only entire solutions to the maximal surface equation

$$
\operatorname{Maximal}[u]=\operatorname{Div}\left(\frac{D u}{\sqrt{1-|D u|^{2}}}\right)=0, \quad|D u|^{2}<1,
$$

are affine functions.

Here the condition $|D u|^{2}<1$ means precisely that the graph defined by $u$ is spacelike.

In this note we show how classical Bernstein's theorem on minimal surfaces in the Euclidean space $\mathbb{R}^{3}$ can be seen as a consequence of Calabi-Bernstein's theorem on maximal surfaces in the Lorentz-Minkowski space $\mathbb{L}^{3}$ (and viceversa). This follows from the following duality between solutions to their corresponding differential equations.

THEOREM 3. Let $\Omega \subseteq \mathbb{R}^{2}$ be a simply connected domain. There exists a non-affine $C^{2}$ solution to the minimal surface equation on $\Omega$

$$
\operatorname{Minimal}[u]=\operatorname{Div}\left(\frac{D u}{\sqrt{1+|D u|^{2}}}\right)=0
$$

if and only if there exists a non-affine $C^{2}$ solution to the maximal surface equation on $\Omega$

$$
\operatorname{Maximal}[w]=\operatorname{Div}\left(\frac{D w}{\sqrt{1-|D w|^{2}}}\right)=0, \quad|D w|^{2}<1 .
$$

\section{PROOF OF THEOREM 3}

Proof. Assume that $u$ is a non-affine solution of Minimal $[u]=0$ on the domain $\Omega$. Recall that for a vector field $X$ on $\mathbb{R}^{2}$ it holds that

$$
(\operatorname{Div} X) d x \wedge d y=d \omega_{J X},
$$

where $J$ denotes the positive $\pi / 2$-rotation in the plane and $\omega_{J X}$ denotes the 1 -form on $\mathbb{R}^{2}$ which is metrically equivalent to the field $J X$, that is, $\omega_{J X}$ satisfies

$$
\omega_{J X}(Y)=\langle J X, Y\rangle
$$

for every vector field $Y$ on $\mathbb{R}^{2}$. Then Minimal $[u]=0$ is equivalent to the fact that $\omega_{J U}$ is closed on $\Omega$, where $U$ is the field on $\Omega$ given by

$$
U=\frac{D u}{\sqrt{1+|D u|^{2}}} .
$$


Then since the domain $\Omega$ is simply connected, we can write

$$
J\left(\frac{D u}{\sqrt{1+|D u|^{2}}}\right)=D w
$$

for a certain $C^{2}$ function $w$ on $\Omega$. Since $J$ is an isometry, there follows

$$
|D w|^{2}=\frac{|D u|^{2}}{1+|D u|^{2}}<1
$$

and also

$$
1+|D u|^{2}=\frac{1}{1-|D w|^{2}} .
$$

From (2), we see that $w$ satisfies the spacelike condition. Besides, using that $J^{2}=-\mathrm{id}$, we obtain from (1) and (3) that

$$
J\left(\frac{D w}{\sqrt{1-|D w|^{2}}}\right)=\sqrt{1+|D u|^{2}} J(D w)=D(-u),
$$

and so Maximal $[w]=0$ follows on $\Omega$.

If $w$ were affine, then $D w$ is a constant vector, $|D w|^{2} \equiv$ constant, and then it follows from (3) that $|D u|^{2}$ is a constant also. It then follows from (1) that $D u$ is a constant vector, contradicting the assumption that $u$ is non-affine.

A very similar argument, starting with a non-affine solution of $\operatorname{Maximal}[w]=0$ on $\Omega$ with $|D w|^{2}<1$, produces a non-affine solution of Minimal $[u]=0$ on $\Omega$.

In particular, when $\Omega$ is the whole plane $\mathbb{R}^{2}$ we obtain the following.

COROLlary 4. There exists an entire, non-affine $C^{2}$ solution to the minimal surface equation

$$
\operatorname{Minimal}[u]=\operatorname{Div}\left(\frac{D u}{\sqrt{1+|D u|^{2}}}\right)=0
$$

on $\mathbb{R}^{2}$ if and only if there exists an entire, non-affine $C^{2}$ solution to the maximal surface equation

$$
\operatorname{Maximal}[w]=\operatorname{Div}\left(\frac{D w}{\sqrt{1-|D w|^{2}}}\right)=0, \quad|D w|^{2}<1
$$

on $\mathbb{R} 2$.

\section{ACKNOWLEDGEMENTS}

This work was written while the first author was visiting the Departamento de Matemática of the Universidade Federal do Ceará, Fortaleza, Brazil. He would like to thank that institution and the members of the department for their wonderful hospitality. This visit was partially supported by FUNCAP, Brazil. L.J. Alías was partially supported by DGICYT and Fundación Séneca (PRIDTYC) CARM, Spain. 


\section{RESUMO}

Nesta nota, mostramos como o clássico teorema de Bernstein sobre as superfícies mínimas no espaço Euclideano pode ser visto como uma consequência do teorema de Calabi-Bernstein sobre as superfícies máximas no espaço de Lorentz-Minkowski (e vice-versa). Isto decorre de uma simples, mas elegante, dualidade entre soluções a suas correspondentes equações diferenciais.

Palavras-chave: Equações de superfícies mínimas, Equações de superfícies máximas, teorema de Bernstein, teorema de Calabi-Bernstein.

\section{REFERENCES}

BERNSTEIN S. 1915-1917. Sur un théoréme de géometrie et ses applications aux équations dérivées partielles du type elliptique, Comm Soc Math Kharkov 15: 38-45.

Calabi E. 1970. Examples of Bernstein problems for some nonlinear equations, Proc Symp Pure Math 15: 223-230. 